\title{
Molecular characterization and pathogenicity of an infectious cDNA clone of tomato brown rugose fruit virus
}

\author{
Ziyue Ma ${ }^{1}$, Hui Zhang ${ }^{2}$, Ming Ding ${ }^{3}$, Zhongkai Zhang ${ }^{3}$, Xiuling Yang ${ }^{1 *}$ (D) and Xueping Zhou ${ }^{1,4^{*}}$
}

\begin{abstract}
Tomato brown rugose fruit virus (ToBRFV) is a new member of the genus Tobamovirus, and has the potential to affect the production and marketability of tomatoes and peppers. In this study, we sequenced and analyzed the complete genome of ToBRFV isolates from tomato plants showing mosaic and mottling symptoms in Yunnan Province of China. We constructed a full-length infectious cDNA clone of ToBRFV, which could induce systemic infection with typical symptoms in tomato, Nicotiana benthamiana, and N. tabacum cv. Samsun nn plants through Agrobacterium-mediated inoculation. Further experimental evidence demonstrated that the rod-shaped virions accumulating in agroinfiltrated plants are sap-transmissible. This is the first report on the construction of a biologically active, full-length infectious cDNA clone of ToBRFV. The system developed herein will facilitate further research on functions of ToBRFV-encoded proteins and plant-ToBRFV interactions through reverse genetic approaches.
\end{abstract}

Keywords: Tobamovirus, Tomato brown rugose fruit virus, Infectious clone, Agroinoculation

\section{Background}

Tomato is one of the world's most cultivated and extensively consumed vegetable crops, with an annual production of about 180 million tons in 2019 (FAOSTAT 2020, http://www.fao.org/faostat/en/\#data). Despite the increased annual production of tomatoes over the past 5 years, the yield and fruit quality of tomato plants are largely affected by an array of pests and diseases during their growing season in the field or postharvest period. Viruses are major disease-causing pathogens that threaten the sustainable production of tomatoes. At present, at least 136 viral species have been reported to infect tomato crops (Hanssen et al. 2010). Tobamovirus is one of the most important genera that has posed a serious threat to tomato production, especially to

\footnotetext{
* Correspondence: yangxiuling@caas.cn; zzhou@zju.edu.cn

'State Key Laboratory for Biology of Plant Diseases and Insect Pests, Institute of Plant Protection, Chinese Academy of Agricultural Sciences, Beijing 100193, China

Full list of author information is available at the end of the article
}

trellised tomato plants grown inside greenhouses, nethouses, or other protected structures.

Tomato brown rugose fruit virus (ToBRFV), a new species of the genus Tobamovirus, was first characterized in 2016 (Salem et al. 2016). As with other members of the genus Tobamovirus, ToBRFV has a positive singlestranded RNA genome that is encapsidated into a rodshaped particle of about $300 \mathrm{~nm}$ in length and $18 \mathrm{~nm}$ in diameter. It can be transmitted by contact, propagation materials, seeds, and bumblebees (Levitzky et al. 2019; Davino et al. 2020). Tomato plants infected with ToBRFV show mosaic and deformation symptoms on leaves, and yellow spots or rough dead patches on tomato fruits, subsequently resulting in severely reduced yield, marketability, and fruit quality (Luria et al. 2017). Although three identified resistance genes, $T m-1, T m-2$, and $T m-2^{2}$, can protect tomato plants from several tobamoviruses such as tomato mosaic virus (ToMV) and tobacco mosaic virus (TMV), they cannot confer resistance to ToBRFV (Luria et al. 2017). While ToBRFV

(c) The Author(s). 2021 Open Access This article is licensed under a Creative Commons Attribution 4.0 International License, which permits use, sharing, adaptation, distribution and reproduction in any medium or format, as long as you give appropriate credit to the original author(s) and the source, provide a link to the Creative Commons licence, and indicate if changes were made. The images or other third party material in this article are included in the article's Creative Commons licence, unless indicated otherwise in a credit line to the material. If material is not included in the article's Creative Commons licence and your intended use is not permitted by statutory regulation or exceeds the permitted use, you will need to obtain permission directly from the copyright holder. To view a copy of this licence, visit http://creativecommons.org/licenses/by/4.0/. 
has spread worldwide in only a few years, its incidence has been reported in Mexico, the United States of America, Germany, Italy, Palestine, Turkey, United Kingdom, Greece, China, Spain, Holland, France, Czech Republic, and Cyprus (Alkowni et al. 2019; Camacho-Beltran et al. 2019; Fidan et al. 2019; Panno et al. 2019; Skelton et al. 2019; Yan et al. 2019; Amer and Mahmoud 2020; Beris et al. 2020; van de Vossenberg et al. 2020; AlfaroFernández et al. 2021).

Infectious clones of plant viruses are important tools for the biological characterization of plant viruses and investigation of virus-host interactions through reverse genetic approaches. Coupled mutagenesis and gene replacement to the infectious clone of plant viruses has promoted our understanding of viral gene expression strategies, the interaction of a virus with its host to complete its life cycle, and resistance responses of plant hosts to combat viral infection. Additionally, virus-based vectors have also been engineered to express foreign genes or induce gene silencing in plants (Abrahamian et al. 2020). Full-length cDNA clones are now available for several species of the genus Tobamovirus, such as TMV, ToMV, and cucumber green mottle mosaic virus (Weber et al. 1992; Chapman 2008; Zheng et al. 2015), facilitating reverse genetic studies of these viruses. However, given the absence of ToBRFV infectious clones, it still remains challenging to perform similar research on this virus.

In this study, we characterized the complete genome sequence of ToBRFV isolates from Yunnan Province of
China. We further constructed a full-length cDNA clone of ToBRFV and evaluated its infectivity in different plants.

\section{Results}

Symptom observation and detection of ToBRFV in tomato plants

During a survey of tomato-infecting viruses, tomato plants showing symptoms typical of tobamovirus infection, such as systemic mosaic and mottling on leaves, were found in Yuanmou County, Yunnan Province of China in October 2020 (Fig. 1a). Thereafter, seven samples were collected and tested for ToBRFV infection by RT-PCR using ToBRFV-specific primers based on the genomic sequences of TMV, ToMV, and ToBRFV. These two primer sets, ToBRFV-591-F/ToBRFV-591-R and ToBRFV-842-F/ToBRFV-842-R, generated amplicons of about 590 and 840-bp, respectively, in all seven samples (Fig. 1b). Among them, five randomly selected 840-bp amplicons (Y2020-3, -4, -5, -6, and -7) were individually cloned and sequenced. Sequence comparisons were performed subsequently, and the alignment result showed $100 \%$ of nucleotide sequence identities among the five isolates, suggesting that they all belong to the same virus species. BLASTn search against the NCBI GenBank database revealed a $99.88 \%$ identity between the 840-bp fragment and the sequence corresponding to the movement protein (MP) gene and coat protein (CP) gene of the ToBRFV-Ant-Tom isolate reported from Turkey (GenBank accession No. a

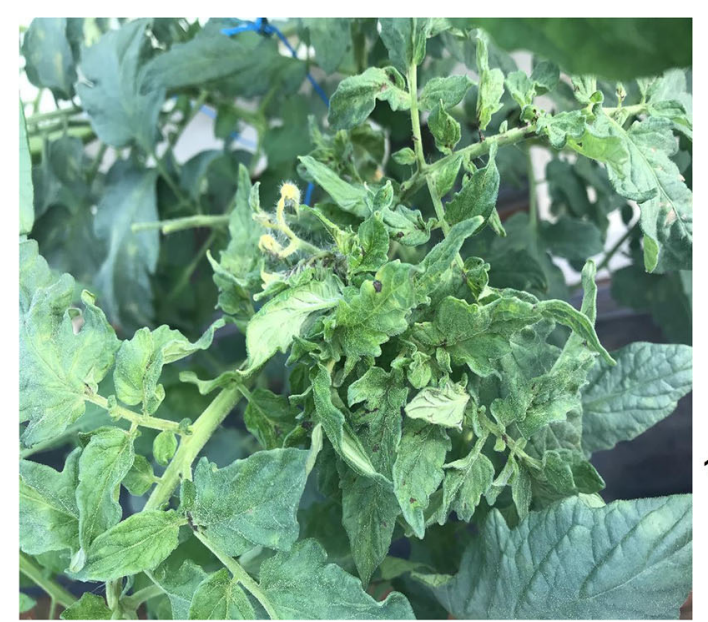

b

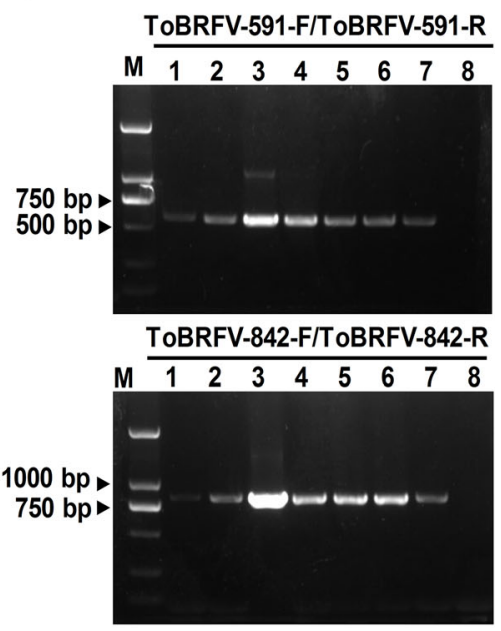

Fig. 1 Detection of tomato brown rugose fruit virus (ToBRFV) from diseased tomato plants. a Symptoms observed on ToBRFV-infected tomato plants in Yunnan Province of China. b Detection of ToBRFV by RT-PCR with two sets of ToBRFV-specific primers. Lane M, DL2000 DNA marker (TaKaRa). Lanes 1-7 indicate PCR products amplified from seven samples (Y2020-1, Y2020-2, Y2020-3, Y2020-4, Y2020-5, Y2020-6, and Y2020-7), respectively. Lane 8 , double distilled water $\left(\mathrm{dd}_{2} \mathrm{O}\right)$ was used as the negative control 
MT107885.1), indicating that the tomato samples collected from Yunnan Province were infected by ToBRFV.

\section{Characterization of the ToBRFV genome and phylogenetic analysis}

To determine the complete nucleotide sequence of ToBRFV Yunnan isolates, total RNA extracted from a representative isolate, Y2020-3, was used for RTPCR analysis. The complete genome sequence of Y2020-3 was determined to be 6390 nucleotides (nts) in length (Accession No. MZ004925). The genome organization of Y2020-3 presented the characteristic features of previously reported ToBRFV isolates, containing four predicted open reading frames (ORFs) (Fig. 2a). The $5^{\prime}$ untranslated region (UTR) is 72 nts in length and rich in A and C residues. The 3' UTR is 209 nts in length. ORF1 and ORF2, separated by a leaky UAG stop codon at position 3423, encode a $126 \mathrm{kDa}$ small replicase subunit and a $183 \mathrm{kDa}$ replicase, respectively. ORF3 and ORF4 encode a 30-kDa MP and a 17.5-

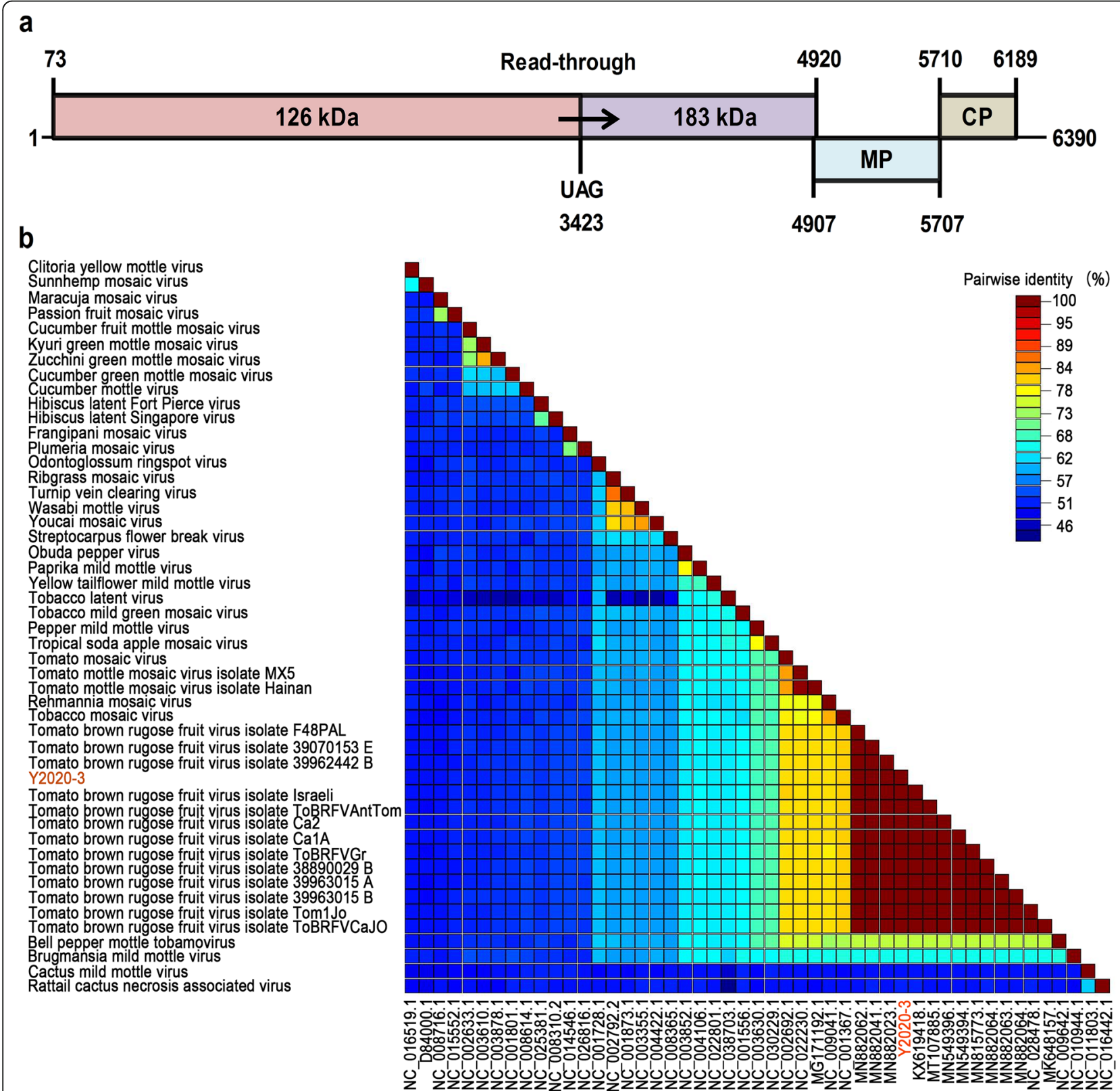

Fig. 2 Genome organization and pairwise identity of ToBRFV. a Diagram depicting the genome organization of ToBRFV. The rectangles correspond to the predicted protein products of the four open reading frames. Positions are marked at the ends. $\mathbf{b}$ Pairwise identity matrix of the complete genome sequences of ToBRFV and other representative tobamoviruses generated using Sequence Demarcation Tool version 1.2 
$\mathrm{kDa} \mathrm{CP}$, respectively (Fig. 2a). The closest relatives of Y2020-3 are two ToBRFV isolates from Israel (ToBRFV-IL, KX619418.1) and Turkey (ToBRFV-AntTom, MT107885.1), presenting 99.84 and $99.83 \%$ nucleotide identities with Y2020-3, respectively. Comparison of the complete genomic sequences of Y2020-3 and ToBRFV-IL identified 12 different nucleotides, two of which were located in the $5^{\prime}$ UTR and the other ten were located within ORF1, ORF2, and ORF3. Among these ten nucleotide variations observed in Y2020-3 and ToBRFV-IL, nine variations did not result in amino-acid changes, whereas, a single variation in ORF1 resulted in an amino-acid change (Ala to $\mathrm{Val}^{1064}$ ). Genomic sequence comparison of Y2020-3 with other tobamovirus species revealed that it shared the highest nucleotide identity of $82.42 \%$ with TMV (Fig. 2b). Phylogenetic analysis of the complete genome sequences of Y2020-3, several ToBRFV isolates and other representative members of the genus Tobamovirus showed that Y2020-3 was grouped in the same cluster with ToBRFV isolates, as expected from their high sequence identities, and separated from other tobamoviruses (Fig. 3). According to the established principles of tobamovirus taxonomy and nomenclature, the isolate was named ToBRFV-[Y2020-3].

\section{Infectivity and pathogenicity of the constructed ToBRFV infectious clone}

To obtain an infectious clone suitable for agroinfection, the full-length genomic sequence of ToBRFV-[Y2020-3] was cloned into the binary vector $\mathrm{pCB} 301$ under the control of a cauliflower mosaic virus $35 \mathrm{~S}$ promoter via a one-step assembly strategy (Fig. 4a). The infectivity and pathogenicity of the full-length cDNA clone of ToBRFV (pCB301-ToBRFV) were tested in tomato (natural host) and four other plant species of the family Solanaceae. At 2 weeks post-inoculation (wpi), typical symptoms such as leaf mottling and narrowing began to appear in the non-inoculated systemic leaves of tomato plants $(n=30)$ infiltrated with Agrobacterium harboring pCB301ToBRFV (Fig. 4b). The leaf narrowing symptoms became much more pronounced at 3-4 wpi. When Nicotiana benthamiana plants were challenged with the infectious clone of ToBRFV, yellowing and leaf curling symptoms developed on the non-inoculated systemic leaves at 6 days post-inoculation (dpi), with necrotic lesions on the inoculated local leaves (Fig. 4b). N. tabacum cv. Samsun nn plants inoculated with the infectious clone of ToBRFV exhibited mosaic symptoms at 2 wpi (Fig. 4b). Viral RNA accumulation in the systemic leaves of agroinoculated tomato, $N$. benthamiana, and $N$. tabacum cv. Samsun nn plants was confirmed by RT-PCR (Fig. 4c). Notably, no visible symptoms or viral RNA accumulation were observed in the corresponding control treatment (tomato, $N$. benthamiana, and $N$. tabacum cv.
Samsun nn plants infiltrated with Agrobacterium harboring pCB301) (Fig. 4b, c), and also in N. tabacum NN and N. tabacum cv. Samsun NN plants agroinoculated with the infectious clone (data not shown). Transmission electron microscopic observation of crude extracts from the symptomatic systemic leaves of tomato and $N$. benthamiana plants revealed the presence of rod-shaped viral particles with an average size of $(250 \pm 50) \mathrm{nm}$ in length and $(18 \pm 5) \mathrm{nm}$ in width (Fig. $4 \mathrm{~d}$ ), representing the typical morphological characteristics of the genus Tobamovirus.

To evaluate whether the virions produced in plants infected with the infectious clone of ToBRFV are biologically active, a mechanical transmission assay was carried out. The crude sap was extracted from the systemic leaves of tomato plants agroinoculated with pCB301ToBRFV, and was then gently rubbed onto the leaves of healthy $N$. benthamiana plants. The systemic leaves of sap-inoculated $N$. benthamiana plants displayed symptoms indistinguishable from those agroinoculated with the infectious clone of ToBRFV (Fig. 5a). Accordingly, RT-PCR analysis demonstrated the presence of viral RNA in the systemic leaves of mechanically inoculated $N$. benthamiana plants (Fig. 5b). These results suggested that viral progeny from agroinoculated tomato plants was readily mechanically transmissible.

\section{Discussion}

ToBRFV poses a continuous and potentially damaging threat to tomato production. In this study, we determined and analyzed the complete nucleotide sequence of ToBRFV isolates from Yunnan Province of China. We also constructed an infectious full-length cDNA clone of ToBRFV, which will facilitate our understanding of the biological processes of ToBRFV and its interaction with hosts.

The occurrence of ToBRFV was first reported in Israel in 2014 (Luria et al. 2017). Since then, the virus spread rapidly and is now widely distributed in the Middle East, North-America, Europe, and Asia (van de Vossenberg et al. 2020). In China, ToBRFV was initially reported to infect tomatoes in Shandong Province in 2019 (Yan et al. 2019). Here, we report the first case of ToBRFV infection on tomato plants in Yunnan Province of China. Sequence comparison and phylogenetic analysis showed that ToBRFV-[Y2020-3] has the closest evolutionary relationship with the Israeli isolate of ToBRFV. For decades, tomato varieties harboring the $T m-2^{2}$ resistance gene exhibited durable resistance to both TMV and ToMV. However, this resistance has been jeopardized by the Israeli isolate of ToBRFV, and systemic infection by ToBRFV was observed in all the six tested tomato cultivars harboring the $T m-2^{2}$ resistance certified by the Tomato Genetic Resource Center (Luria et al. 2017). 


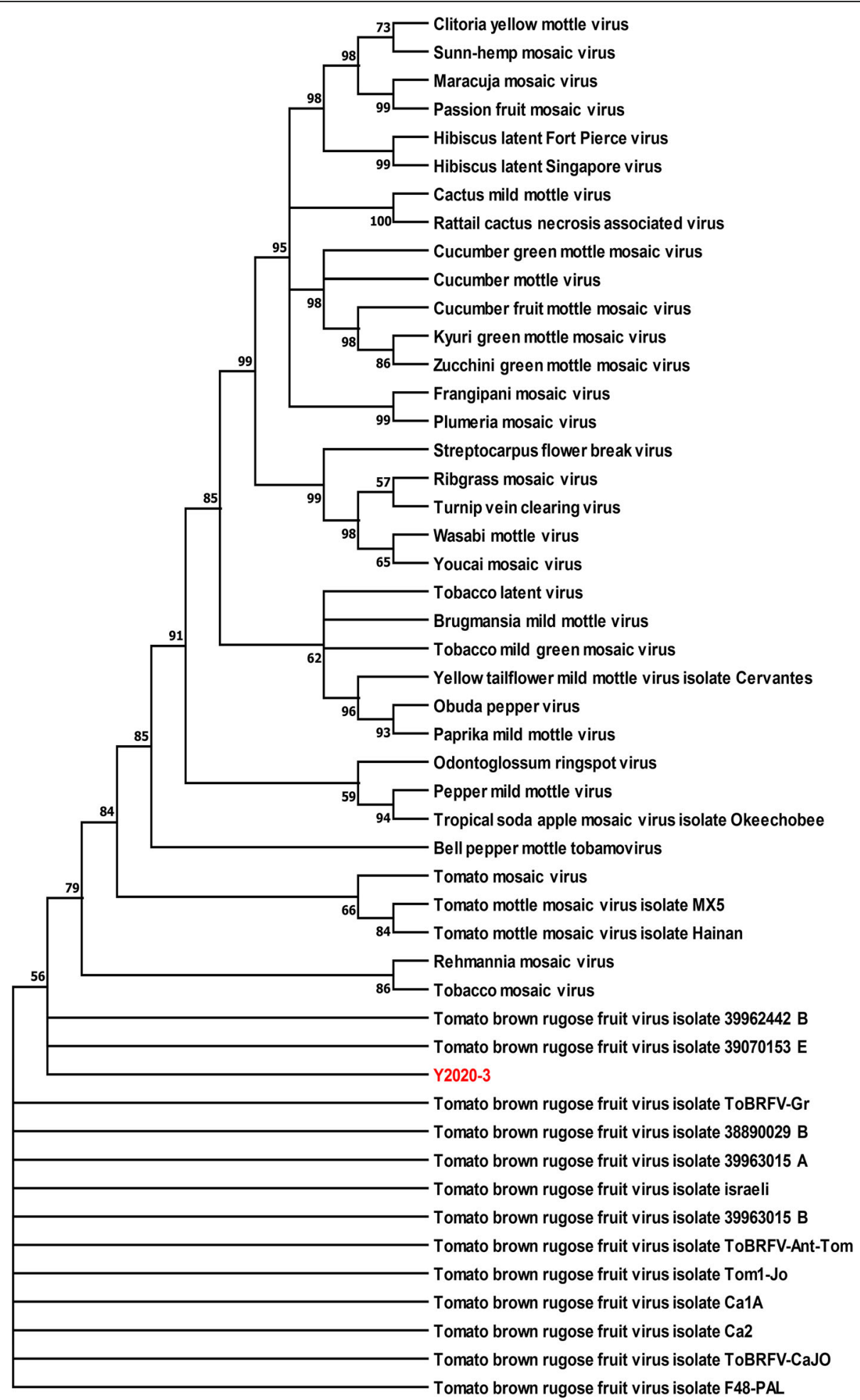

Fig. 3 Neighbor-joining phylogenetic tree constructed based on the complete genome sequences of ToBRFV and the representative tobamoviruses. The statistical significance of the branches was calculated by bootstrap with 1000 replicates. Branches with less than $50 \%$ bootstrap support have been collapsed 
a

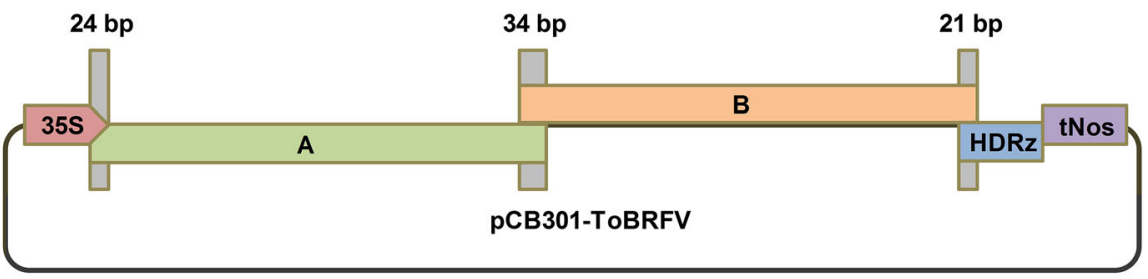

b Solanum lycopersicum

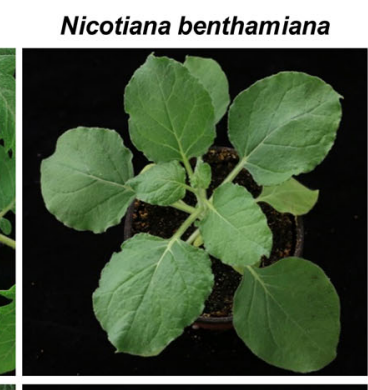

Nicotiana tabacum cv.
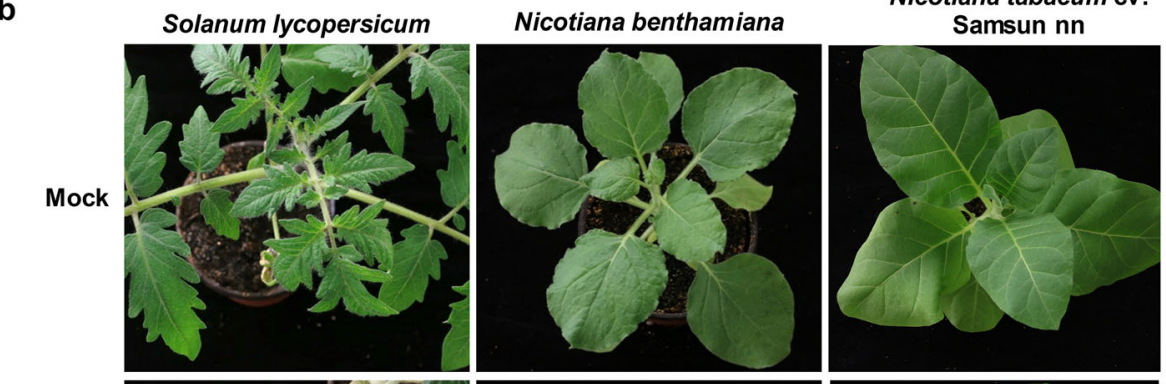

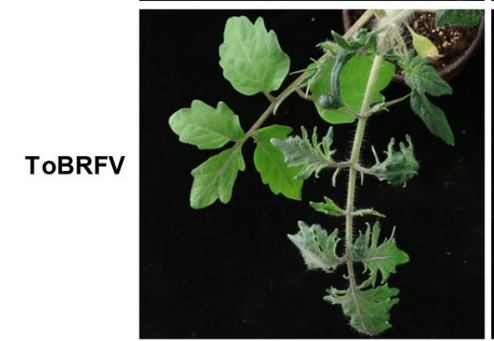

C

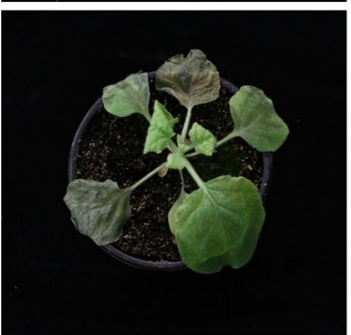

d

ToBRFV-F-1534/ToBRFV-R-3733
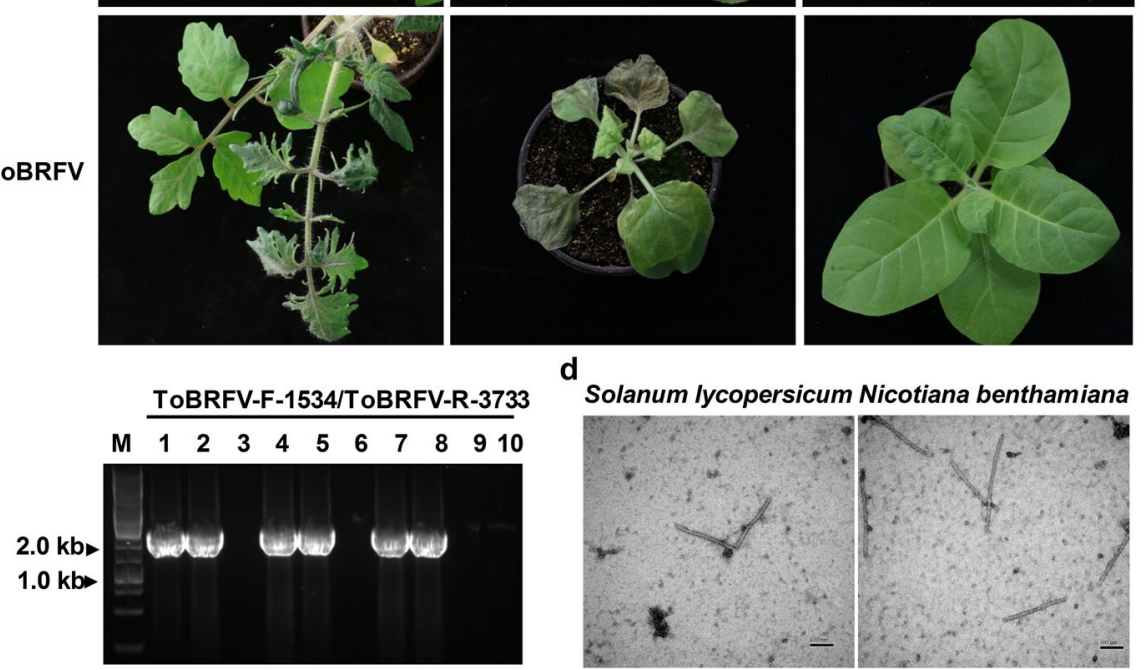

Fig. 4 Infectivity and pathogenicity of the infectious clone of ToBRFV. a Schematic diagram illustrating the one-step assembly cloning strategy used for the construction of an infectious full-length cDNA clone of ToBRFV. Two overlapping ToBRFV fragments, A and B, were amplified by polymerase chain reaction and infused to the linearized pCB301 vector. The overlapping regions were illustrated by gray rectangles, showing the number of overlapping nucleotides. 35S, the cauliflower mosaic virus promoter; HDRz, hepatitis delta virus ribozyme ribozyme; tNos, Nos terminator. b Symptoms in tomato (Solanum lycopersicum), Nicotiana benthamiana, and N. tabacum cv. Samsun nn plants induced by ToBRFV infection. In the upper panel, plants were mock-inoculated with agrobacteria harboring the pCB301 vector. In the lower panel, plants were agroinoculated with the infectious clone of ToBRFV (pCB301-ToBRFV). Images were taken at $21 \mathrm{dpi}$ for tomato plants, and $14 \mathrm{dpi}$ for $\mathrm{N}$. benthamiana and N. tabacum cv. Samsun nn plants. c RT-PCR analysis of ToBRFV RNA accumulation in systemic leaves of agroinoculated plants using ToBRFV-specific primers. Lane M, GeneRuler 1 kb DNA ladders (Thermo); lanes 1-2, RNA extracted from N. benthamiana plants agroinoculated with pCB301-ToBRFV; lanes 4-5, RNA extracted from tomato plants agroinoculated with pCB301-ToBRFV; lanes 7-8, RNA extracted from N. tabacum cv. Samsun nn plants agroinoculated with pCB301-ToBRFV. Lanes 3, 6, and 9, represent RNA extracted from mock-inoculated N. benthamiana, tomato, and N. tabacum cv. Samsun nn plants, respectively. Lane 10, dd $\mathrm{H}_{2} \mathrm{O}$ (negative control). d Transmission electron micrograph of viral particles in systemic leaves of tomato and $\mathrm{N}$. benthamiana plants agroinoculated with pCB301-ToBRFV. Bar $=100 \mathrm{~nm}$

Likewise, systemic infection by ToBRFV was also observed in all the 50 tested commercial tomato cultivars in China (Yan et al. 2021), though it is not known whether the tested tomato cultivars harbor the $T m-2^{2}$ resistance gene. China ranks first in tomato production in the world (FAOSTAT, http://www.fao.org/faostat/en/ \#data). Since the damage caused by ToBRFV is continuously spreading in China, more attention should be paid to the occurrence and epidemiology of ToBRFV. In addition, serious efforts are required to reduce the incidence of infection with ToBRFV and consequent economic losses by screening and breeding ToBRFVresistant tomato cultivars.

Owing to its strong infectivity, ToBRFV has been reported in several countries. However, due to the short history of discovery, the functions of ToBRFV-encoded 
a
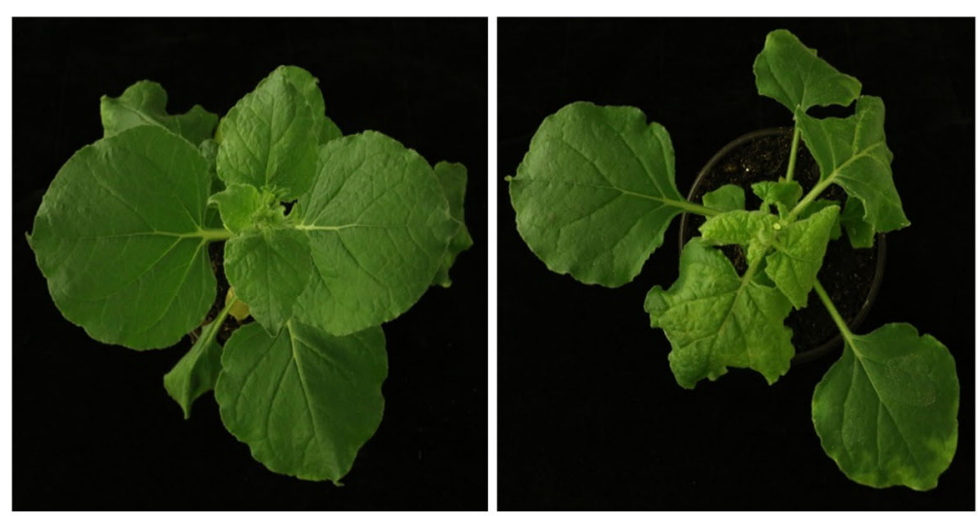

b
ToBRFV-591-F/ToBRFV-591-R

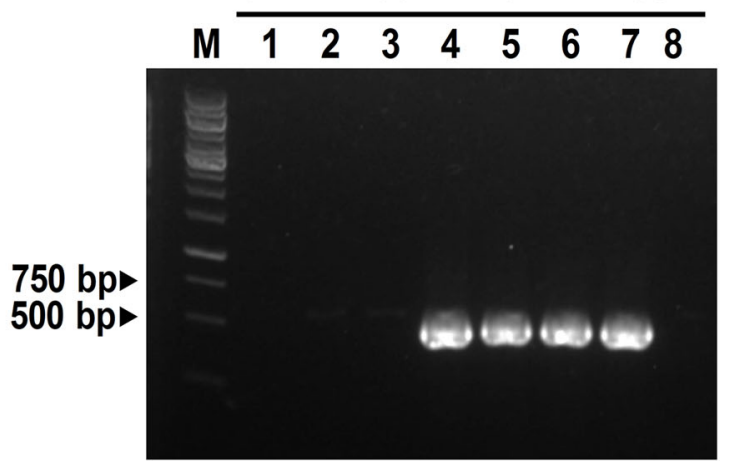

Fig. 5 Sap transmission of ToBRFV particles produced in tomato plants infected with the infectious clone of ToBRFV. a Symptom of $N$. benthamiana plant mechanically inoculated with crude sap extracted from systemic leaves of tomato plants agroinoculated with the ToBRFV infectious clone at $7 \mathrm{dpi}$. b RT-PCR detection of ToBRFV RNA accumulation in systemic leaves of mechanically inoculated $N$. benthamiana plants using ToBRFV-specific primers. Lane M, GeneRuler 1 kb DNA ladders (Thermo); lanes 1-3, samples mock-inoculated with PBS buffer; lanes 4-7, samples collected from systemic leaves of mechanically inoculated $\mathrm{N}$. benthamiana plants; lane $8, \mathrm{ddH}_{2} \mathrm{O}$ (negative control)

proteins are still unknown. Plant virus infectious clones provide powerful tools to investigate the functions of viral proteins and virus-host interactions. Our data show that the full-length cDNA clone of ToBRFV constructed in this study can establish systemic infection by Agrobacterium-mediated inoculation. With the successful construction of the ToBRFV infectious clone, we can now use reverse genetics to study ToBRFV genes governing various functions such as virus replication, movement, symptom development, and resistance-breaking.

\section{Conclusions}

In this study, we characterized ToBRFV isolates from Yunnan Province, one of the main tomato-producing provinces in China. We found that the ToBRFV isolate is most closely related to an Israeli isolate (ToBRFV-IL). Moreover, the construction and characterization of the ToBRFV infectious clone will serve as an important tool to employ reverse genetic approaches to advance our current knowledge about the functions of ToBRFV- encoded proteins, and the interactions of ToBRFV with its plant hosts.

\section{Methods}

Plant materials and growth conditions

Tomato plants showing mosaic symptoms were collected from Yuanmou County, Yunnan Province of China in October 2020. Solanum lycopersicum cv. Moneymaker, $N$. benthamiana, N. tabacum NN, N. tabacum cv. Samsun NN, and N. tabacum cv. Samsun nn plants used for virus inoculation were grown in an insect-free growth room at $25^{\circ} \mathrm{C}$ under a $16 \mathrm{~h}$ light $/ 8 \mathrm{~h}$ dark cycle.

RNA extraction, reverse transcription, and virus detection Total RNA was extracted from symptomatic leaves with TRIzol reagent following the manufacturer's standard protocol (Invitrogen, Carlsbad, CA, USA). First-strand cDNA was synthesized with $1 \mu \mathrm{g}$ of total RNA using Prime-Script $^{\text {TM }}$ II cDNA Synthesis Kit and random primers (TaKaRa, Japan). Reverse transcription was 
performed according to the manufacturer's recommended protocols. Two ToBRFV-specific primer sets, ToBRFV-591-F/R and ToBRFV-842-F/R, which were expected to amplify fragments of 591 and $842 \mathrm{bps}$, respectively, were individually used for molecular detection of ToBRFV. The primer sequences are listed in Table 1. The polymerase chain reaction (PCR) was performed with TransStart $^{\circ}$ FastPfu high-fidelity DNA polymerase (TransGen, Beijing, China).

\section{Complete genome amplification and sequencing}

To obtain the complete nucleotide sequence of the ToBRFV Yunnan isolate, the genome data of ToBRFV deposited in the NCBI GenBank database were used to design primers for RT-PCR analysis. Four primers sets, ToBRFV-F-23/ToBRFV-R-1623, ToBRFV-F-1534/ ToBRFV-R-3733, ToBRFV-F-3669/ToBRFV-R-4720, and ToBRFV-F-4570/ToBRFV-R-5717 (Table 1), were designed to amplify adjacent regions with overlapping

Table 1 Primers used for detection and amplification of tomato brown rugose fruit virus

\begin{tabular}{|c|c|}
\hline $\begin{array}{l}\text { Primer } \\
\text { name }\end{array}$ & Primer sequences $\left(5^{\prime} \rightarrow 3^{\prime}\right)$ \\
\hline ToBRFV-591-F & GACAGGTGAATGGAATTTGCCAGATAATTG \\
\hline ToBRFV-591-R & AGACATATTTAATACGAATCTGAATCGGCG \\
\hline ToBRFV-842-F & GAAGTCCCGATGTCTGTAAGG \\
\hline ToBRFV-842-R & GTGCCTACGGATGTGTATGA \\
\hline ToBRFV-F-23 & CAACAACAACAAACAACAAACAACA \\
\hline $\begin{array}{l}\text { ToBRFV-R- } \\
1623\end{array}$ & CTAATGCGTCTCCCGACAC \\
\hline $\begin{array}{l}\text { ToBRFV-F- } \\
1534\end{array}$ & AGATTTCCCTGGCTTTTGGA \\
\hline $\begin{array}{l}\text { ToBRFV-R- } \\
3733\end{array}$ & ATCATCGCCACCAAATTTC \\
\hline $\begin{array}{l}\text { ToBRFV-F- } \\
3669\end{array}$ & ATGGTACGAACGGCGGCAG \\
\hline $\begin{array}{l}\text { ToBRFV-R- } \\
4720\end{array}$ & CAATCCTTGATGTGTTTAGCAC \\
\hline $\begin{array}{l}\text { ToBRFV-F- } \\
4570\end{array}$ & ACCTTATGTGGAATTTCGAGG \\
\hline $\begin{array}{l}\text { ToBRFV-R- } \\
5717\end{array}$ & AGACATATTTAATACGAATCTGAATCGGCG \\
\hline 5' RACE-GSP1 & GATTACGCCAAGCTTCACCCTGCCTGTGTTTCCACCTGCTG \\
\hline 3'RACE-GSP2 & $\begin{array}{l}\text { GATTACGCCAAGCTTATGACGCAACGGTGGCTATAAGGAG } \\
\text { CGC }\end{array}$ \\
\hline $\begin{array}{l}\text { pCB-TOBRFV- } \\
1 \mathrm{~F}\end{array}$ & $\begin{array}{l}\text { AGTTCATTTCATTTGGAGAGGCCTGTATTIITACAACATAT } \\
\text { ACCAA }\end{array}$ \\
\hline $\begin{array}{l}\text { pCB-ToBRFV- } \\
1 \mathrm{R}\end{array}$ & CCTCGAGAAAGCAGCGCCTCTITATCAGACTGA \\
\hline $\begin{array}{l}\text { pCB-ToBRFV- } \\
2 \mathrm{~F}\end{array}$ & CTCAGTCTGATAAAGAGGCGCTGCTTTCTCGAGGATAT \\
\hline $\begin{array}{l}\text { pCB-ToBRFV- } \\
2 R\end{array}$ & $\begin{array}{l}\text { GAGATGCCATGCCGACCCGGGTGGGCCCCTACCGGGGG } \\
\text { TTC }\end{array}$ \\
\hline
\end{tabular}

ends. PCR amplification was carried out with each of the primer sets using TransStart ${ }^{\circ}$ FastPfu high-fidelity DNA polymerase (TransGen). The PCR products were purified with Gel Extraction Kit (Omega, Norcross, GA, USA), cloned into the pEasy-Blunt vector (TransGen), and sequenced by Sanger sequencing. Sequences were edited and assembled using Lasergene 7.0 (Madison, WI, USA). The $5^{\prime}$ and $3^{\prime}$ gene-specific primer (GSP), 5' RACE-GSP1, and 3' RACE-GSP2 were subsequently designed based on the obtained partial sequence of ToBRFV. The missing 5' - and 3'-terminal sequences of the ToBRFV genome were obtained by performing $5^{\prime}$ and 3' rapid amplification of cDNA ends (RACE), respectively, using SMARTer RACE cDNA Amplification Kit (Clontech, CA, USA), and were cloned into the pRACE vector for subsequent Sanger sequencing. Two clones from each amplicon were sequenced to confirm the sequence. The complete nucleotide sequence was assembled and analyzed with the software Lasergene 7.0 and submitted to GenBank under accession number (MZ004925).

\section{Sequence analysis}

The complete nucleotide sequence of the ToBRFV Yunnan isolate was compared with those of ToBRFV available in GenBank database (http://www.ncbi.nlm.nih.gov) using the BLASTn program. Multiple sequence alignment was carried out using Clustal X implemented in MEGA version 7.0. A phylogenetic tree derived from a nucleotide sequence alignment of ToBRFV and the representative tobamoviruses was constructed using the neighbor-joining method with 1000 bootstrap replicates by MEGA 7.0 (Kumar et al. 2016). Sequence pairwise identity was analyzed with Sequence Demarcation Tool version 1.2 (SDTv1.2) using the Clustal W model (Muhire et al. 2014).

\section{Construction of the infectious clone of ToBRFV}

The infectious clone of ToBRFV was constructed using a one-step assembly strategy as shown in Fig. 4a. In brief, primers were designed based on the complete sequence of ToBRFV Yunnan isolate and the plant binary vector pCB301, with 21-34 bp overlapping nucleotides at fragment ends. Primer sets pCB-ToBRFV-1F/pCBToBRFV-1R and pCB-ToBRFV-2F/pCB-ToBRFV-2R (Table 1) were used to amplify fragment $\mathrm{A}$ and $\mathrm{B}$ of ToBRFV, respectively. RT-PCR and PCR reactions were performed using TransStart $^{\circ}$ FastPfu high-fidelity DNA polymerase (TransGen) with cDNA of the Y2020-3 isolate as template. The pCB301 vector was linearized with FastDigest restriction enzymes SmaI and StuI (Thermo Fisher, USA). PCR products and the linearized pCB301 were gel purified with Gel Extraction Kit (Omega). The overlapping DNA fragments were seamlessly assembled 
to the linearized pCB301 vector using infusion recombinase (ClonExpress II One Step Cloning Kit, Vazyme, Nanjing, China). Three independent clones for each assembled recombinant were sequenced. The resultant recombinant plasmid pCB301-ToBRFV was then introduced into Agrobacterium tumefaciens EHA105 by electroporation.

\section{Agroinoculation of plants}

Agroinoculation of plants was performed as described by Yang et al. (2018). Briefly, A. tumerfaciens strain EHA105 containing the full-length cDNA sequence of ToBRFV was incubated in LB broth supplemented with $100 \mu \mathrm{g} / \mathrm{mL}$ of kanamycin and $40 \mu \mathrm{g} / \mathrm{mL}$ of rifampicin overnight at $28^{\circ} \mathrm{C}$. After centrifugation at $8000 \times g$ for $5 \mathrm{~min}$, the agrobacteria cells were collected and resuspended with infiltration buffer (10 mM MgCl, $10 \mathrm{mM}$ MES (pH 5.8), $100 \mu \mathrm{M}$ acetosyringone) to an $\mathrm{OD}_{600}$ of 1.0 , and incubated at room temperature for $3 \mathrm{~h}$. For agroinoculation of tomato plants, a 1-mL fine syringe was used to inject agrobacteria suspension into the stems and abaxial leaves of tested plants at the four-leaf stage. For agroinoculation of $N$. benthamiana and $N$. tabacum plants, agrobacteria suspension was infiltrated into the abaxial surface of plant leaves using a 1 -mL needleless syringe.

\section{Transmission electron microscopic observation of viral particles}

About $0.1 \mathrm{~g}$ of ToBRFV-infected systemic leaf tissues of $N$. benthamiana and tomato plants were homogenized in $1 \mathrm{~mL}$ of $0.01 \mathrm{~mol} / \mathrm{L}$ phosphate buffer (PBS, $0.01 \mathrm{~mol} / \mathrm{L}$ $\left.\mathrm{KH}_{2} \mathrm{PO}_{4}: 0.01 \mathrm{~mol} / \mathrm{L} \quad \mathrm{Na}_{2} \mathrm{HPO}_{4}=49: 51 \quad(\mathrm{v} / \mathrm{v}), \mathrm{pH}=7.0\right)$. After centrifugation at $11,300 \times \mathrm{g}$ for $15 \mathrm{~min}$, the supernatant was fixed in $2.5 \%$ glutaraldehyde overnight at $4{ }^{\circ} \mathrm{C}$. Ten $\mu \mathrm{L}$ of the supernatant and $10 \mu \mathrm{L}$ of $1 \%$ uranyl acetate were placed together onto the copper grids of 300 mesh covered with a membrane (Fidan et al. 2020). The droplets were mixed and incubated at room temperature for $3 \mathrm{~min}$. Excess liquid was removed from the edge of the copper mesh with a filter paper, and viral particles were observed under a transmission electron microscope (Hiltachi, H-7650) at a voltage of $80 \mathrm{kV}$.

\section{Mechanical transmission of ToBRFV}

The ToBRFV-infected systemic leaf tissues $(0.5 \mathrm{~g})$ of tomato plants were homogenized in $5 \mathrm{~mL}$ of $0.01 \mathrm{M}$ PBS buffer. The crude sap was gently rubbed onto celitedusted surface of $N$. benthamiana plant leaves at four to six-leaf stage. $N$. benthamiana plants mechanically inoculated with PBS served as negative controls. After inoculation, plants were maintained in a clean growth chamber at $25^{\circ} \mathrm{C}$ under a photoperiod of 16 -h light/8-h dark, and were observed daily for symptoms development. Virus detection was performed at 14 dpi by RTPCR as described above.

\section{Abbreviations}

aa: Amino acid; bp: Base pair; CP: Coat protein; GSP: Gene-specific primer; MP: Movement protein; ORF: Open reading frame; PBS: Phosphate buffer; RACE: Rapid amplification of cDNA ends; RT-PCR: Reverse transcriptionpolymerase chain reaction; TMV: Tobacco mosaic virus; ToBRFV: Tomato brown rugose fruit virus; ToMV: Tomato mosaic virus; UTR: Untranslated region

\section{Acknowledgments}

We would like to thank Prof. Xiaorong Tao from Nanjing Agricultural University for providing the pCB301 vector.

\section{Authors' contributions}

$X Y$ and $X Z$ designed the research; $Z M, X Y, X Z, H Z, M D$, and $Z Z$ performed the experiments; $Z M$ and $X Y$ analyzed the data; $Z M, X Y$, and $X Z$ wrote the paper. All authors read and approved the final manuscript.

\section{Funding}

This work was supported by grants from National Natural Science

Foundation of China (31972245 and 31720103914).

Availability of data and materials

Not applicable.

\section{Declarations}

Ethics approval and consent to participate

Not applicable.

\section{Consent for publication}

Not applicable.

\section{Competing interests}

The authors declare that they have no competing interests.

\section{Author details}

${ }^{1}$ State Key Laboratory for Biology of Plant Diseases and Insect Pests, Institute of Plant Protection, Chinese Academy of Agricultural Sciences, Beijing 100193, China. ${ }^{2}$ Horticultural Research Institute, Shanghai Academy of Agriculture Sciences, Shanghai 201106, China. ${ }^{3}$ Yunnan Province Key Laboratory of Agricultural Biotechnology, Institute of Biotechnology and Germplasm Resources, Yunnan Academy of Agricultural Sciences, Kunming 650223, China. ${ }^{4}$ State Key Laboratory of Rice Biology, Institute of Biotechnology, Zhejiang University, Hangzhou 310058, China.

Received: 25 April 2021 Accepted: 23 June 2021

Published online: 05 July 2021

\section{References}

Abrahamian P, Hammond RW, Hammond J. Plant virus-derived vectors: applications in agricultural and medical biotechnology. Ann Rev Virol. 2020; 7(1):513-35. https://doi.org/10.1146/annurev-virology-010720-054958.

Alfaro-Fernández A, Castillo P, Sanahuja E, Rodríguez-Salido MC, Font MI. First report of tomato brown rugose fruit virus in tomato in Spain. Plant Dis. 2021; 105(2):515. https://doi.org/10.1094/PDIS-06-20-1251-PDN.

Alkowni R, Alabdallah O, Fadda Z. Molecular identification of tomato brown rugose fruit virus in tomato in Palestine. J Plant Pathol. 2019;101(3):719-23. https://doi.org/10.1007/s42161-019-00240-7.

Amer MA, Mahmoud SY. First report of tomato brown rugose fruit virus on tomato in Egypt. New Dis Rep. 2020;41(1):24. https://doi.org/10.5197/j.20440588.2020.041.024.

Beris D, Malandraki I, Kektsidou O, Theologidis I, Vassilakos N, Varveri C. First report of tomato brown rugose fruit virus infecting tomato in Greece. Plant Dis. 2020;104(7):2035. https://doi.org/10.1094/PDIS-01-20-0212-PDN.

Camacho-Beltran E, Perez-Villarreal A, Leyva-Lopez NE, Rodriguez-Negrete EA, Ceniceros-Ojeda EA, Mendez-Lozano J. Occurrence of tomato brown rugose fruit virus infecting tomato crops in Mexico. Plant Dis. 2019;103(6):1440. https://doi.org/10.1094/PDIS-11-18-1974-PDN

Chapman SN. Construction of infectious clones for RNA viruses: TMV. In: Foster GD, Johansen IE, Hong Y, Nagy PD, editors. Plant virology protocols. Methods 
in molecular biology ${ }^{\mathrm{TM}}$, vol 451. Totowa: Humana Press; 2008. https://doi. org/10.1007/978-1-59745-102-4_32.

Davino S, Caruso AG, Bertacca S, Barone S, Panno S. Tomato brown rugose fruit virus: seed transmission rate and efficacy of different seed disinfection treatments. Plants. 2020;9(11):1615. https://doi.org/10.3390/plants9111615.

Fidan H, Sarikaya P, Calis O. First report of tomato brown rugose fruit virus on tomato in Turkey. New Dis Rep. 2019;39(1):18. https://doi.org/10.5197/j.20440588.2019.039.018.

Fidan H, Sarikaya P, Yildiz K, Topkaya B, Erkis G, Calis O. Robust molecular detection of the new tomato brown rugose fruit virus in infected tomato and pepper plant from Turkey. J Integr Agr. 2020;19:2-11.

Hanssen IM, Lapidot M, Thomma BPHJ. Emerging viral diseases of tomato crops. Mol Plant-Microbe Interact. 2010;23(5):539-48. https://doi.org/10.1094/ MPMI-23-5-0539.

Kumar S, Stecher G, Tamura K. MEGA7: molecular evolutionary genetics analysis version 7.0 for bigger datasets. Mol Biol Evol. 2016;33(7):1870-4. https://doi. org/10.1093/molbev/msw054

Levitzky N, Smith E, Lachman O, Luria N, Mizrahi Y, Bakelman H, et al. The bumblebee Bombus terrestris carries a primary inoculum of tomato brown rugose fruit virus contributing to disease spread in tomatoes. PLoS One. 2019;14(1):e0210871. https://doi.org/10.1371/journal.pone.0210871.

Luria N, Smith E, Reingold V, Bekelman I, Lapidot M, Levin I, et al. A new Israeli tobamovirus isolate infects tomato plants harboring $T m-22$ resistance genes. PLoS One. 2017;12(1):e0170429. https://doi.org/10.1371/journal.pone.0170429.

Muhire BM, Varsani A, Martin DP. SDT: a virus classification tool based on pairwise sequence alignment and identity calculation. PLoS One. 2014;9(9):e108277. https://doi.org/10.1371/journal.pone.0108277.

Panno S, Caruso AG, Davino S. First report of tomato brown rugose fruit virus on tomato crops in Italy. Plant Dis. 2019;103(6):1443. https://doi.org/10.1094/ PDIS-12-18-2254-PDN.

Salem N, Mansour A, Ciuffo M, Falk BW, Turina M. A new tobamovirus infecting tomato crops in Jordan. Arch Virol. 2016;161(2):503-6. https://doi.org/10.1 007/s00705-015-2677-7.

Skelton A, Buxton-Kirk A, Ward R, Harju V, Frew L, Fowkes A, et al. First report of tomato brown rugose fruit virus in tomato in the United Kingdom. New Dis Rep. 2019;40(1):12. https://doi.org/10.5197/j.2044-0588.2019.040.012.

van de Vossenberg BTLH, Visser M, Bruinsma M, Koenraadt HMS, Westenberg M, Botermans M. Real-time tracking of tomato brown rugose fruit virus (ToBRFV) outbreaks in the Netherlands using Nextstrain. PLoS One. 2020;15(10): e0234671. https://doi.org/10.1371/journal.pone.0234671.

Weber $\mathrm{H}$, Haeckel P, Pfitzner AJP. A cDNA clone of tomato mosaic virus is infectious in plants. J Virol. 1992;66(6):3909-12. https://doi.org/10.1128/jvi.66. 6.3909-3912.1992

Yan ZY, Ma HY, Han SL, Geng C, Tian YP, Li XD. First report of tomato brown rugose fruit virus infecting tomato in China. Plant Dis. 2019;103(11):2973. https://doi.org/10.1094/PDIS-05-19-1045-PDN.

Yan ZY, Zhao MS, Ma HY, Liu LZ, Yang GL, Geng C, et al. Biological and molecular characterization of tomato brown rugose fruit virus and development of quadruplex RT-PCR detection. J Integr Agr. 2021;20(7):1871-9. https://doi. org/10.1016/S2095-3119(20)63275-0.

Yang X, Ren Y, Sun S, Wang D, Zhang F, Li D, et al. Identification of the potential virulence factors and RNA silencing suppressors of mulberry mosaic dwarfassociated geminivirus. Viruses. 2018;10(9):472. https://doi.org/10.3390/v1 0090472.

Zheng H, Xiao C, Han K, Peng J, Lin L, Lu Y, et al. Development of an agroinoculation system for full-length and GFP-tagged cDNA clones of cucumber green mottle mosaic virus. Arch Virol. 2015;160(11):2867-72. https://doi.org/10.1007/s00705-015-2584-y.

Ready to submit your research? Choose BMC and benefit from:

- fast, convenient online submission

- thorough peer review by experienced researchers in your field

- rapid publication on acceptance

- support for research data, including large and complex data types

- gold Open Access which fosters wider collaboration and increased citations

- maximum visibility for your research: over $100 \mathrm{M}$ website views per year

At $\mathrm{BMC}$, research is always in progress.

Learn more biomedcentral.com/submissions 